\title{
Problema APSP via Dimensão-VC e Médias de Rademacher
}

\author{
Alane M. de Lima ${ }^{1}$, André L. Vignatti ${ }^{1}$, Murilo V. G. da Silva ${ }^{1}$ \\ ${ }^{1}$ Departamento de Informática - Universidade Federal do Paraná (UFPR) \\ Caixa Postal 19.081 - Curitiba - PR - Brazil \\ \{amlima, vignatti,murilo\}einf.ufpr.br
}

\begin{abstract}
We consider the version of the All-pairs Shortest Paths (APSP) problem, where we are only required to compute paths with high centrality, such that the centrality metric reflects the "importance" of a path in the graph. We propose an algorithm for this problem that uses a sampling approach based on $V C$-Dimension and Rademacher averages. In the case of sparse graphs with logarithmic diameter, which is a common model for several real-world scenarios, the sample size is exponentially smaller than those obtained by classical techniques (e.g. Hoeffding and union bound).
\end{abstract}

Resumo. Considere o problema de computar o caminho mínimo entre cada par de vértices (APSP) de um grafo, desde que o caminho tenha alta centralidade, sendo que a métrica de centralidade em questão reflete a "importância" do caminho no grafo. Propomos um algoritmo para este problema que usa uma estratégia de amostragem baseada em Dimensão-VC e médias de Rademacher. No caso de grafos esparsos de diâmetro logarítmico, que comumente modelam situações reais, o tamanho da amostra é exponencialmente menor do que aquelas obtidas por técnicas clássicas (e.g.: Hoeffding e limitante da união).

\section{Introdução}

O caminho mínimo entre todos os pares de vértices de um grafo (All-pairs Shortest Paths - APSP) é um problema amplamente estudado. Na prática, entretanto, computá-lo de maneira exata torna-se inviável em grafos de larga escala. Neste artigo propomos um algoritmo de amostragem de tempo esperado $\mathcal{O}\left(\lg \operatorname{Diam}_{V}(G) \max (m+\right.$ $\left.n \log n, \operatorname{Diam}_{V}(G)^{2}\right)$ ), onde $\operatorname{Diam}_{V}(G)$ é a maior quantidade de vértices em um caminho mínimo de um grafo $G$ (diâmetro em vértices de $G$ ) de $n$ vértices e $m$ arestas. O algoritmo computa um caminho mínimo exato $P$ entre todo par de vértices $(u, v)$ se a centralidade de $P$ (uma medida de "importância" de $P$ ) for maior do que uma constante fixa. Intuitivamente, quanto mais caminhos mínimos tiverem $P$ como subcaminho, maior sua centralidade. Usando técnicas desenvolvidas por [Riondato e Upfal 2018], aplicamos a teoria de Dimensão Vapnik-Chervonenkis (Dimensão-VC) e médias de Rademacher em um algoritmo de amostragem progressiva para provar que amostrar no máximo $\left\lceil\frac{c}{\epsilon}\left\lfloor 2 \lg \operatorname{Diam}_{V}(G)+1\right\rfloor \ln \left(\frac{1}{\epsilon}\right)+\ln \frac{1}{\delta}\right\rceil$ caminhos mínimos (e inspecionar seus subcaminhos) é suficiente para encontrar com probabilidade $1-\delta$ todos os caminhos mínimos de centralidade mínima $\epsilon$ ( $c$ é aproximadamente $\frac{1}{2}$ ). Observamos que o limitante para o tamanho da amostra obtido neste artigo é exponencialmente menor do que se fosse alcançado por meio de técnicas tradicionais como os limitantes da união e de Hoeffding, por exemplo. 
Os algoritmos exatos mais eficientes para o problema APSP são propostos por [Williams 2014] (grafos com pesos em $\mathbb{N}$ ), de tempo $\mathcal{O}\left(\frac{n^{3}}{2^{c \sqrt{\log n}}}\right)$ para alguma constante $c>0$, e por [Pettie e Ramachandran 2002] (grafos com pesos em $\mathbb{R}^{+}$), de tempo $\mathcal{O}(m n \log \alpha(m, n))$, onde $\alpha(m, n)$ é a função de Ackermann inversa. Observe que em comparação a tais trabalhos nosso algoritmo é mais eficiente em grafos densos, e no pior caso, atinge o tempo do algoritmo de [Pettie e Ramachandran 2002] no caso de grafos esparsos. Contudo, em grafos esparsos com diâmetro logarítmico (cenário presente em diversas aplicações reais [Easley e Kleinberg 2010]), nosso algoritmo é melhor do que o de tais autores para o nosso problema, alcançando o tempo esperado de $O(n \log n \log \log n)$.

\section{Preliminares}

Seja $G=(V, E)$ um grafo ponderado não-direcionado e $n=|V|$ e $m=|E|$. S.p.d.g. assumimos $G$ conexo. Uma árvore de caminhos mínimos de $u \in V$ é uma árvore geradora $T_{u}$ de $G$ onde todo caminho em $T_{u}$ de $u$ para qualquer $v \in V$ é um caminho mínimo $p_{u v}$ em $G$. Fixamos uma ordenação arbitrária de $V$ e consideramos $T_{u}$ como uma árvore retornada pelo algoritmo de Dijkstra, denominando-a árvore Dijkstra. Um ramo $\mathcal{B}_{u}(v)$ de $T_{u}$ é um caminho $p_{u v}$ com início na raiz de $T_{u}$ e destino em $v(u \neq v)$. Cada subcaminho de $\mathcal{B}_{u}(v)$ é também um caminho mínimo em $G$, e denotamos tal conjunto de subcaminhos (incluindo $p_{u v}$ ) por $S\left(p_{u v}\right)$ ou $S\left(\mathcal{B}_{u}(v)\right)$ (por conveniência, ambas as notações são usadas). Como $G$ é não-direcionado, então $p_{v u}$ também é um caminho mínimo em $G$ e $S\left(p_{v u}\right)$ denota todos os seus subcaminhos (incluindo $p_{v u}$ ).

Definição 1 (Centralidade de Caminho Mínimo). Dados $G=(V, E),(u, v) \in V^{2} e T_{a}$ $\forall a \in V$, a centralidade de caminho mínimo de $(u, v)$ é $c(u, v)=\frac{t_{u v}}{n(n-1)}$ onde $t_{u v}=$ $\sum_{(a, b) \in V^{2}: a \neq b} \mathbb{1}_{\tau_{u v}}\left(\mathcal{B}_{a}(b)\right), \tau_{u v}=\left\{\mathcal{B}_{c}(d) \in \bigcup_{a \in V} \bigcup_{b \in V: b \neq a} \mathcal{B}_{a}(b): p_{u v} \in S\left(\mathcal{B}_{c}(d)\right)\right\}$.

A Dimensão- $V C$ fornece limitantes superiores à complexidade de amostras, isto é, o tamanho mínimo que uma amostra deve ter para que parâmetros de erro e confiança sejam atingidos, baseada na estrutura do espaço de intervalos que modela um determinado problema. Contudo, encontrar limitantes justos para o tamanho fixo de uma amostra pode ser uma tarefa complexa. Neste caso, uma alternativa é usar um algoritmo baseado em amostragem progressiva, isto é, que aumenta a amostra iterativamente enquanto o parâmetro do limitante máximo para o erro não é atingido. A ideia central consiste no fato que a condição de parada (quando a amostra é grande o suficiente) é baseada em Médias de Rademacher, um conceito que reside no núcleo de teoria de aprendizado estatístico.

Um espaço de intervalos é um par $\mathcal{R}=(U, \mathcal{I})$ onde $U$ é um domínio (finito ou infinito) e $\mathcal{I}$ é uma coleção de subconjuntos de $U$ (intervalos). Dado $S \subseteq U$, a projeção de $\mathcal{I}$ em $S$ é o conjunto $\mathcal{I}_{S}=\{S \cap I \mid I \in \mathcal{I}\}$. Dizemos que $S$ é despedaçado por $\mathcal{I}$ se $\left|\mathcal{I}_{S}\right|=2^{|S|}$. A Dimensão-VC de $\mathcal{R}=(U, \mathcal{I})$ é $\operatorname{VCDim}(\mathcal{R})=\max \{k: \exists S \subseteq$ $U$ tal que $|S|=k$ e $\left.\left|\mathcal{I}_{S}\right|=2^{k}\right\}$. De modo geral, para um conjunto de valores de interesse $\mathcal{H}$, existe uma família de funções $\mathcal{F}$ de $U$ a $\mathbb{R}^{*}$ tal que há uma $f_{h} \in \mathcal{F}, \forall h \in \mathcal{H}$. Seja $S=\left(z_{1}, \ldots, z_{r}\right)$ uma coleção de $r$ elementos de $U$ amostrados com respeito a $\pi$. Então $\forall f_{h} \in \mathcal{F}, L_{S}\left(f_{h}\right)=(1 / r) \sum_{s \in S} f_{h}(s)$ e $L_{U}\left(f_{h}\right)=\mathbb{E}_{u \in U}\left[f_{h}(u)\right]$.

Teorema 1 ([Har-Peled e Sharir 2011]). Dados $\mathcal{R}=(U, \mathcal{I}) \operatorname{com} \operatorname{VCDim}(\mathcal{R}) \leq k$, uma distribuição de probabilidades $\pi$ em $U, 0<\epsilon, \delta<1$ e $c>0$ : 
1. $\operatorname{Pr}\left(\left|L_{S}\left(f_{h}\right)-L_{U}\left(f_{h}\right)\right| \leq \epsilon\right) \geq 1-\delta, \forall f_{h} \in \mathcal{F}$, para uma coleção de elementos $S \subseteq U$ amostrados segundo $\pi$ tal que $|S|=\left(c / \epsilon^{2}\right)(k+\ln (1 / \delta))$.

2. $\operatorname{Pr}\left(|I \cap S| \geq 1\right.$ se $\left.\operatorname{Pr}_{\pi}(I) \geq \epsilon\right) \geq 1-\delta, \forall I \in \mathcal{I}$, para uma coleção de elementos $S \subseteq U$ amostrados segundo $\pi$ tal que $|S|=(c / \epsilon)(k \ln 1 / \epsilon+\ln (1 / \delta))$.

Definição 2. A média empírica de Rademacher de uma família de funções $\mathcal{F}$ com respeito a $S$ e uma dist. $\sigma$ de $r$ variáveis aleatórias de Rademacher com $\operatorname{Pr}\left(\sigma_{i}=1\right)=\operatorname{Pr}\left(\sigma_{i}=\right.$ $-1)=1 / 2(1 \leq i \leq r)$ é definida como $\tilde{R}_{r}(\mathcal{F}, S)=\mathbb{E}_{\sigma}\left[\sup _{f_{h} \in \mathcal{F}}(1 / r) \sum_{i=1}^{r} \sigma_{i} f_{h}\left(z_{i}\right)\right]$.

Teorema 2 ([Oneto et al. 2013]). $\sup _{f_{h} \in \mathcal{F}}\left|L_{S}\left(f_{h}\right)-L_{U}\left(f_{h}\right)\right| \leq 2 \tilde{R}_{r}(\mathcal{F}, S)+\ln \frac{3}{\delta}+$ $\left(\sqrt{\left(\ln \frac{3}{\delta}+4 r \tilde{R}_{r}(\mathcal{F}, S)\right) \ln \frac{3}{\delta}}\right) / r+\sqrt{\ln \left(\frac{3}{\delta}\right) /(2 r)}$ com probabilidade pelo menos $1-\delta$.

Teorema 3 ([Riondato e Upfal 2018]). Considere $v_{f_{h}}=\left(f_{h}\left(z_{1}\right), \ldots, f_{h}\left(z_{r}\right)\right)$ em uma amostra $S=\left\{z_{1}, \ldots, z_{r}\right\},|S|=r, e \mathcal{V}_{S}=\left\{v_{f_{h}}, f_{h} \in \mathcal{F}\right\}$. Então $\tilde{R}_{r}(\mathcal{F}, S) \leq$ $\min _{s \in \mathbb{R}^{+}} w(s)$, onde $w(s)=\frac{1}{s} \ln \sum_{v_{f_{h}} \in \mathcal{V}_{S}} \exp \left(s^{2}\left\|v_{f_{h}}\right\|_{2}^{2} /\left(2 r^{2}\right)\right)$.

\section{Algoritmo Proposto}

Nesta seção definimos $c(u, v)$ em termos de um espaço de intervalos e dados $0<\delta, \epsilon<1$, apresentamos o Algoritmo 1 que retorna as distâncias entre caminhos com $c(u, v) \geq \epsilon$ (tabela $d$ ) de $G$, com probabilidade $1-\delta$. A tabela $d$ contém as distâncias que foram computadas pelo algoritmo. Além disso, são retornadas as estimativas da centralidade entre cada par de vértices (tabela $\tilde{c}$ ), se assim for desejado. A atualização da tabela de árvores canônicas $\tilde{t}$ e do conjunto $\mathcal{V}$ (que contém os valores de $\tilde{t}$ sem repetição) é feita de modo análogo ao Algoritmo 2 em [Riondato e Upfal 2018]. Dado um cronograma de crescimento de amostra $\left(\left|S_{i}\right|\right)_{i \geq 1}$, seja $\mathcal{T}$ o conjunto de $n$ árvores Dijkstra de $G$; temos que $\mathcal{H}=V^{2}$ e $U=\bigcup_{a \in V} \bigcup_{b \in V: b \neq a} \mathcal{B}_{a}(b)$, e $\forall(u, v) \in V^{2}, \mathcal{I}=\left\{\tau_{u v}:(u, v) \in V^{2}\right\}$. Para cada $\mathcal{B}_{a}(b) \in U$, seja a função $f_{u v}: U \rightarrow\{0,1\}$ definida como $f_{u v}\left(\mathcal{B}_{a}(b)\right)=\mathbb{1}_{\tau_{u v}}\left(\mathcal{B}_{a}(b)\right)$. Temos que $\mathcal{F}=\left\{f_{u v}:(u, v) \in V^{2}\right\}$. Cada $\mathcal{B}_{a}(b)$ é amostrado com probabilidade $\frac{1}{n(n-1)}$ em $U$ e $\mathbb{E}\left[f_{u v}\left(\mathcal{B}_{a}(b)\right)\right]=L_{U}\left(f_{u v}\right)=c(u, v), \forall(u, v) \in V^{2}$. Seja $S=\left\{\mathcal{B}_{a_{i}}\left(b_{i}\right), 1 \leq i \leq r\right\}$ um conjunto de $r$ ramos amostrados independentemente em $U$. A estimativa da centralidade é $\tilde{c}(u, v)=L_{S}\left(f_{u v}\right)=\frac{1}{r} \sum_{\mathcal{B}_{a_{i}}\left(b_{i}\right) \in S} f_{u v}\left(\mathcal{B}_{a_{i}}\left(b_{i}\right)\right)$. Por argumentos semelhantes ao Teorema 4.1 em [de Lima et al. 2020], provamos um limitante superior para $\operatorname{VCDim}(\mathcal{R})$. A corretude e o limite superior para o tempo de execução do algoritmo são demonstrados nos Teoremas 5 e 6 . Por limitações de espaço, as provas dos lemas enunciados nesta seção serão omitidas neste trabalho e apresentadas em sua respectiva versão estendida.

Teorema 4. $\operatorname{VCDim}(\mathcal{R}) \leq\left\lfloor 2 \lg \operatorname{Diam}_{V}(G)+1\right\rfloor$.

$\mathrm{Na}$ amostragem progressiva, sejam $S_{1}$ e $\delta_{1}$ os valores iniciais do tamanho da amostra e de $\delta$, respectivamente. Então $S_{1} \geq\left(\ln (6 / \delta)\left(1+\sqrt{1+8^{2} \epsilon^{2}}\right)\right) /\left(4 \epsilon^{2}\right)$ (aplicação do Teorema 2 considerando $\delta_{1}=\delta / 2, \tilde{\mathcal{R}}_{r}\left(\mathcal{F}, S_{1}\right) \geq 0$ e um limite superior de $\left.\epsilon\right)$. Em cada iteração $i, S_{i}=g^{i} S_{1}, \forall i \geq 1$, para uma constante $g>1$.

Teorema 5. Considere $S_{r} \subseteq U$ de tamanho $r$, e seja $\eta_{i}$ o valor de $\eta$ na $i$-ésima iteração do laço 2-11. Então $\eta_{r}=2 w_{s}+\ln \frac{3}{\delta_{r}}+\sqrt{\left(\ln \frac{3}{\delta_{r}}+4\left|S_{r}\right| w_{s}\right) \ln \frac{3}{\delta_{r}}} /\left|S_{r}\right|+\sqrt{\ln \frac{3}{\delta_{r}} /\left(2\left|S_{r}\right|\right)}$, onde $\delta_{r}=\delta / 2^{r}$, é o valor em que $r=\min \{i \geq 1\}$ tal que $\eta_{r} \leq \epsilon$ para o grafo $G=$ $(V, E)$ e constantes $0<\epsilon, \delta<1$. O Algoritmo 1 retorna com probabilidade $1-\delta$ a distância exata $d(u, v), \forall(u, v) \in V^{2}$, tal que $d(u, v)>0$ se $c(u, v) \geq \epsilon$, e garante $\operatorname{Pr}(|\tilde{c}(u, v)-c(u, v)| \leq \epsilon) \geq 1-\delta$. 
Teorema 6. Dado $G=(V, E)$, o Algoritmo 1 tem tempo esperado $\mathcal{O}\left(\lg \operatorname{Diam}_{V}(G)\right.$. $\left.\max \left(m+n \log n, \operatorname{Diam}_{V}(G)^{2}\right)\right)$ para computar d e $\tilde{c}$ em uma amostra de tamanho $r=\left\lceil\frac{c}{\epsilon}\left(\left\lfloor 2 \lg \operatorname{Diam}_{V}(G)+1\right\rfloor \ln \frac{1}{\epsilon}+\ln \frac{1}{\delta}\right)\right\rceil$ e $r=\left\lceil\frac{c}{\epsilon^{2}}\left(\left\lfloor 2 \lg \operatorname{Diam}_{V}(G)+1\right\rfloor+\ln \frac{1}{\delta}\right)\right\rceil$, respectivamente.

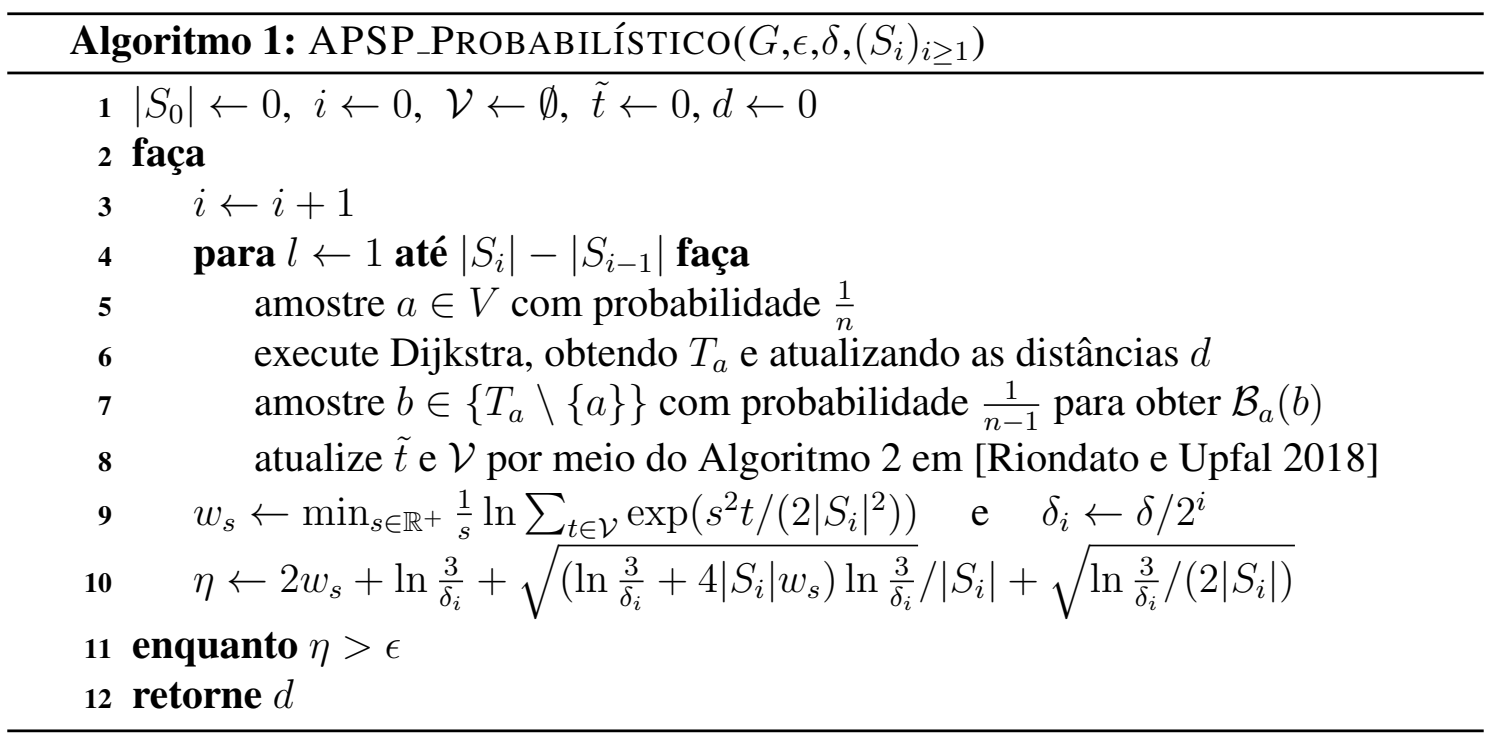

\section{Considerações Finais}

Apresentamos um algoritmo baseado em amostragem que executa em tempo esperado $\mathcal{O}\left(\lg \operatorname{Diam}_{V}(G) \cdot \max \left(m+n \log n, \operatorname{Diam}_{V}(G)^{2}\right)\right)$ que retorna a distância exata entre $(u, v)$ com probabilidade $1-\delta$ se a centralidade de caminho mínimo de $(u, v)$ é pelo menos $\epsilon$. No caso de grafos esparsos de diâmetro logarítmico (presentes em diversas aplicações reais), nosso algoritmo executa em tempo esperado $O(n \log n \log \log n)$.

\section{Referências}

de Lima, A. M., da Silva, M. V. G., e Vignatti, A. L. (2020). Estimating the percolation centrality of large networks through pseudo-dimension theory. ACM SIGKDD Conference on Knowledge Discovery and Data Mining (KDD).

Easley, D. e Kleinberg, J. (2010). Networks, crowds, and markets. Cambridge Un. Press.

Har-Peled, S. e Sharir, M. (2011). Relative (p, $\varepsilon$ )-approximations in geometry. Discrete \& Computational Geometry, 45(3):462-496.

Oneto, L., Ghio, A., Anguita, D., e Ridella, S. (2013). An improved analysis of the rademacher data-dependent bound using its self bounding property. Neural Networks, 44:107 - 111 .

Pettie, S. e Ramachandran, V. (2002). Computing shortest paths with comparisons and additions. In Proc. of ACM-SIAM Symp. on Discrete Algorithms, pages 267-276.

Riondato, M. e Upfal, E. (2018). Abra: Approximating betweenness centrality in static and dynamic graphs with rademacher averages. ACM Transactions on Knowledge Discovery from Data, 12(5):61:1-61:38.

Williams, R. (2014). Faster all-pairs shortest paths via circuit complexity. In Proc. of the Forty-sixth Annual ACM Symp. on Theory of Computing, STOC '14, pages 664-673. 